\title{
Visual and Analytic Strategies in Geometry
}

\author{
George Kospentaris $^{a}$, Stella Vosniadou ${ }^{b}$,Smaragda Kazic, Emilian Thanou ${ }^{\mathrm{d}}$ \\ ${ }^{a}$ National and Kapodistrian University of Athens, Greece \\ ${ }^{\mathrm{b}}$ The Flinders University of South Australia, Australia and National and Kapodistrian University of Athens, Greece \\ ${ }^{\mathrm{c}}$ Panteion University of Social and Political Sciences, Greece \\ ${ }^{\mathrm{d}}$ National and Kapodistrian University of Athens, Greece
}

Article received 13 November / revised 28 December / accepted 19 January / available online 3 March

\begin{abstract}
We argue that there is an increasing reliance on analytic strategies compared to visuospatial strategies, which is related to geometry expertise and not on individual differences in cognitive style. A Visual/Analytic Strategy Test (VAST) was developed to investigate the use of visuo-spatial and analytic strategies in geometry in 30 mathematics teachers and $13411^{\text {th }}$ grade students. Students' performance in the VAST was also compared to performance in tests of visuo-spatial abilities, of abstract reasoning, and of geometrical knowledge. The results showed high performance of all the participants in the VAST items that could be solved by relying on visuo-spatial strategies. However, only the math teachers showed high performance in the VAST items that required the application of analytic geometrical strategies. There were high correlations between the students' performance in the tests of visuo-spatial and abstract reasoning abilities and the VAST Analytic Strategies scale, but the contribution of these tests to the VAST analytic performance became statistically insignificant when geometrical knowledge was used as a mediating factor. The implications of this work for the learning and assessment of geometrical knowledge are discussed.
\end{abstract}

Keywords: Geometry learning and instruction; visual-spatial reasoning; analytic strategies; assessment of geometry

Corresponding author: George Kospentaris, ${ }^{a}$ National and Kapodistrian University of Athens, Dimitsanas Str. 20, 11522, Athens, Greece, Email: 25aris@math.uoa.gr DOI: http://dx.doi.org/10.14786/flr.v4i1.226 


\section{Introduction}

In recent years research has accumulated showing that spatial thinking is central to success in science, technology, engineering, and mathematics, the so-called STEM disciplines. Spatial thinking is thinking about the location of objects and their relations and requires both visuo-spatial ability - the ability to mentally visualize the rotation of objects - and spatial abstract reasoning - the ability to identify analogical relations amongst patterns (see Newcombe, 2010; Wai, Lubinski, \& Benbow, 2009). There is convincing evidence that there are important individual differences in spatial thinking and that spatial thinking abilities can predict success in STEM disciplines (Hegarty \& Waller, 2006; Wai, et al., 2009). Particularly impressive are the analyses of large data sets showing that people with high scores on tests of spatial thinking in high school are more interested in science and math, are more likely to get advanced degrees in STEM, and are more likely to pursue STEM careers (Shea, Lubinski, \& Benbow, 2001; Wai et al., 2009). This has led to an increase in training studies that aim at improving spatial thinking as a means of improving performance in STEM disciplines (Sanchez, 2012; Uttal et al., 2013).

There is little doubt that much of the problem solving done in science, mathematics and engineering requires the use of spatial thinking (Kozhevnikov, Motes, \& Hegarty, 2007; Stieff, 2007, Zazkis, Dubinsky, \& Dautermann, 1996). In Euclidean geometry, where figures are the main objects of study, the role of spatial thinking is of utmost importance. Visualizing the shapes and their relation is a standard prerequisite for the understanding of geometrical propositions (Battista, 2007). Although a great deal of this spatial thinking can be achieved using visuo-spatial strategies - i.e., strategies that allow individuals to obtain spatial information from immediate perceptual processes - spatial thinking can also be achieved using analytic strategies, where rules provide access to spatial information without recourse to visual perception and mental animation (Stieff, 2007). Zazkis et al. (1996) showed that the majority of the university students participating in abstract algebra courses used a combination of visuo-spatial and analytic strategies (see also Schwartz \& Black, 1996; Stieff, 2007).

The use of visuo-spatial vs. analytic strategies has been predominately examined from an individual differences point of view, as an individual characteristic or a cognitive style (Eisenberg \& Dreyfus, 1991; Pitta-Pantazi \& Christou, 2009). This emphasis on individual differences has obscured the fact that reliance on analytic strategies also characterizes the acquisition of expertise in many domains of STEM. As expertise is acquired, problem solving increasingly relies on specialized, domain-specific, rule-based, analytic approaches compared to visual, perceptual information and mental rotation. For example, in the domain of organic chemistry expert chemists develop a predilection for analytic strategies to solve chemistry tasks (Stieff, 2007).

In geometry, reliance on visuo-spatial strategies seems to coincide with the Level 1 of the van Hiele (1986) theory in geometrical thinking. At later levels geometrical thinking increasingly requires an understanding of the logical systems that geometry represents. In geometry, shapes are represented by a set of properties and their relations and geometrical thinking is characterized by the formal manipulation of a logical system. Thus, when geometrical expertise is achieved, geometrical thinking relies increasingly on analytical formal processes based on geometrical knowledge. The purpose of the present research is to develop a task that can differentiate visual from analytic reasoning in geometry -- a Visual/Analytic Strategy Task (VAST) -- and to validate it by comparing novices to experts in geometry. In the next section we present a summary review of the literature on geometrical thinking and define and explain our theoretical position with respect to the use of visuo-spatial and analytic strategies.

\subsection{Geometrical thinking}

Piaget and his collaborators (Piaget, Inhelder, \& Szeminska, 1948/1960; Piaget \& Inhelder, 1948/1967) were the first to study the psychological foundations of geometrical thinking and to propose that it develops in four sequential and hierarchical stages ${ }^{1}$. In subsequent years, van Hiele (1986) argued that

\footnotetext{
${ }^{1}$ There is a great deal of research on spatial development in young children and different theoretical approaches have appeared after Piaget's seminal work (see Newcombe \& Huttenlocher, 2000; Spelke \& Kinzler, 2007) but this is not the focus of the present paper. 
there are five, qualitatively distinct, hierarchical levels of thought in geometry. In contrast to Piaget, van Hiele strongly emphasized the crucial role of school instruction in the acquisition of geometrical knowledge (van Hiele, 1986, p. 65-66). More recently, Houdement and Kuzniak (2003) proposed (on theoretical grounds) that the five van Hiele levels can be reduced to three Kuhnian-like paradigms: Geometry 1-Natural Geometry; Geometry II- Natural Axiomatic Geometry, and Geometry III - Formalist Axiomatic Geometry.

Empirical research so far has failed to confirm the predictions of the van Hiele theory that students move through discrete levels of geometrical thought, each characterized by different internal conceptual organization (Battista, 2007). It appears instead that students oscillate between different levels of geometric understanding depending on the context and the nature of the problems to be solved. For this reason some researchers have argued that although there might be different levels of geometric thinking as identified by van Hiele, these do not represent distinct stages but develop in parallel and without discontinuities between them (Clements \& Battista, 2001; Lehrer, Jenkins, \& Osana, 1998).

It follows from the above that we need a theoretical framework that can account for the considerable conceptual re-organizations that take place in the process of acquiring and using geometrical knowledge without posing the existence of hierarchical and well-defined distinct stages. For these reason, it is proposed here that it might be fruitful to examine geometrical thinking from a conceptual change point of view, and that the framework theory (FT) approach to conceptual change (Vosniadou, 2013; Vosniadou \& Skopeliti, 2014) can serve as an anchor for examining changes in geometrical knowledge after exposure to instruction. The FT belongs to a class of conceptual change approaches known as 'theory-theory' (Carey, 2009), but also differs from them in important ways. Briefly, the FT claims that (a) there are systems of core cognition that bootstrap cognitive development (Carey, 2009; Spelke \& Kinzler, 2007), without making strong nativist interpretations of early infant competencies ${ }^{2}$, and (b) that conceptual development consists of episodes of qualitative change, which, however, are not discontinuous or stage like. Rather, conceptual change is seen as a slow and gradual learning process greatly facilitated by sociocultural and educational inputs. According to the FT, the same constructive-type mechanisms that are involved in all learning processes are also involved in conceptual change processes often producing fragmentation and misconceptions, but eventually having the potential to lead to qualitatively different conceptual organizations (Vosniadou \& Skopeliti, 2014). Finally, the FT claims that initial systems of thought continue to exist and influence thinking, even after instruction-induced conceptual changes have occurred (Shtulman \& Varcarcel, 2012; Vosniadou et al., 2015).

Seen from this theoretical perspective, it is argued that geometrical knowledge is originally built on two core cognitive systems (spatial and numerical) that rely on visuo-spatial information (Newcombe \& Frick, 2010; Spelke, Lee, \& Izard, 2010), but that it gradually develops through systematic instruction to rely on more analytic strategies based on formal geometrical knowledge. In other words, we claim that there is a growing reliance on analytic strategies in geometric thinking with the acquisition of expertise, and that the systematic use of analytic strategies is a product of conceptual changes that take place in the subject-matter area of geometry. We do not claim that visuo-spatial strategies become extinct and that experts rely on analytic strategies only. Unlike stage theories, we argue that the initial, visuo-spatial, approach to geometry is not supplanted by the analytic one, but continues to exist and to be used when contextually appropriate. The ability to systematically employ analytic strategies in geometry, however, is a major intellectual achievement and not a matter of individual differences in cognitive style. It is the product of a conceptual change which takes place over many years and which requires the acquisition of new concepts and new forms of geometrical thinking.

We believe that many geometry education researchers would agree with this account of the development of geometrical knowledge. Geometry is undeniably a formal system and geometric reasoning

\footnotetext{
${ }^{2}$ Various theories are attempting to explain early spatial development including connectionist interpretations and neoconstructivist approaches (see Newcombe, Uttal, \& Sauer, in press, for an extensive review).
} 


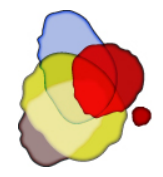

consists of using this formal system to reason about shape and space. According to Battista (2007), underlying this formal system is a 'primitive' system of visuo-spatial thinking allowing individuals to 'see', inspect, and reflect on spatial objects, images, relationships and transformations (p. 843). This 'primitive' system is characterized by what he calls 'perceptual objects' - i.e., mental entities perceived by an individual when viewing physical objects in the real world, including geometrical diagrams. In contrast, expert geometrical knowledge operates on 'conceptual objects' - i.e., abstract, completely idealized and general mental entities based on 'formal' categories, which are explicitly circumscribed according to verbally stated, property-based definitions. The difference between a geometric diagram and a figure captures this basic dichotomy: the former is a material entity, a concrete case that imperfectly represents the abstract concept, while the latter is a theoretical, ideal object without any physical properties. Similarly, Fischbein (1993) argues that experts in geometry form and reason with 'figural concepts'. A figural concept is controlled by logical rules in the context of an axiomatic system but is also a mental entity, an image with a spatial-figural content, although devoid of any concrete sensorial properties (Fischbein, 1993, p. 148).

Battista's (2007) and Fischbein's (1993) arguments are consistent with the proposal that there are ontological and representational shifts that take place in the development of geometrical knowledge analogous in some respects to the ontological shifts that take place in learning science (Chi, 2008; Vosniadou, 2013). For example, a circle, this quite familiar shape, changes from a visual gestalt (Figure 1a) and becomes the locus of all plane points characterized with the property that they are equidistant from its center (Figure 1b), or, in the conceptual frame of analytic geometry, to an equation (the plane points satisfying $x^{2}+y^{2}=r^{2}$, Figure 1c). In addition, the theoretical explanations in the domain also change. At the beginning, geometrical propositions are mainly inductive generalizations based on empirical observations and experimentation with perceptual objects and not on proofs and deductive procedures based on accepted axioms and previously proven propositions.

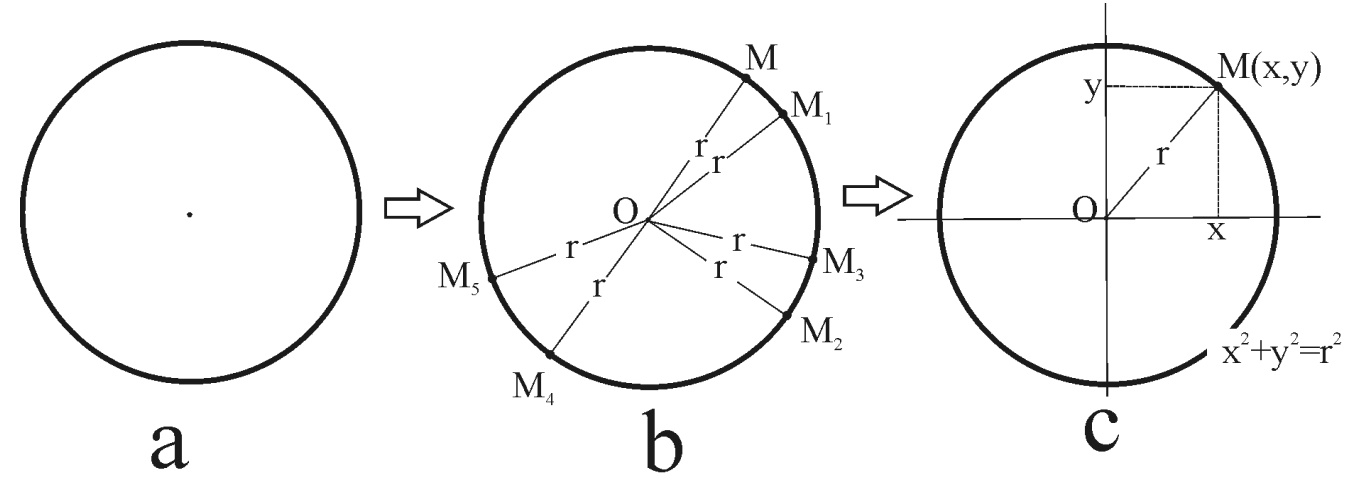

Figure 1. Changes in the representation of the circle.

It could be argued that the above arguments would also be acceptable by stage theories, such as the van Hiele theory. If this is the case, then what can the FT offer in our theoretical understanding of geometrical expertise? Although some stage theories allow for intra-individual differences across tasks (a phenomenon known as decalage -Piaget \& Inhelder, 1948/1967), they nevertheless assume that a) the new forms of thinking that develop in geometry gradually transform and eventually replace the 'primitive' visuospatial system with a more advanced system of thought based on analytical, formal knowledge, and b) that this process leads to distinct, qualitatively different stages in students' thinking. From the perspective of the FT, however, knowledge acquisition does not proceed through hierarchical and well defined distinct stages, but through the gradual assimilation of the new information into the initial, 'primitive' system, creating in the process inert knowledge, fragmentation, and misconceptions, many of which are 'synthetic' conceptions ${ }^{3}$

\footnotetext{
3 Synthetic conceptions are formed when learners assimilate scientific information to their incompatible prior knowledge producing in the process an alternative, erroneous conception, which however has some internal consistency and explanatory value, such as the 'impetus misconception' in mechanics (Clement, 1982), the 'molecules in matter' model in the atomic-molecular theory (Wiser \& Smith, 2013), and the 'hollow sphere' model in observational
} 
(Vosniadou \& Skopeliti, 2014). Although there is an order of acquisition in this conceptual development and some learning progressions can be identified, these cannot be characterized as 'stages', both because they cannot be clearly identified as such, and because the 'primitive' - visuo-spatially-based system - is not eradicated but continues to co-exist with the formal, analytical modes of thought (Shtulman \& Valcarcel, 2012).

The purpose of the present research is not to test a full-blown theory of geometrical thinking, but to start in this direction by developing a valid task that can help us distinguish the use of visuo-spatial from analytic strategies in geometry. In the next section the rationale behind the development of the Visual/Analytic Strategy Task is described.

\subsection{The Visual/Analytic Strategy Task (VAST).}

Several tasks have been developed over the years to test students' movement from the visual to the descriptive/analytic van Hiele level (e.g., Burger \& Shaughnessy, 1986; Gutiérrez \& Jaime, 1998; Lynn \& Lynch, 2010; Usiskin, 1982). The main limitation of such tasks is that they either favour the recall or recognition of definitions of shapes and their properties over their understanding and their application in novel situations, or that they do not require thinking based on more sophisticated, relational properties (Battista (2007).

In addition to the above, there are several other standardized geometry tests that assess students' level of geometrical knowledge, such as the California Standards Geometry Test (CSGT, 2009). These standardized tests examine mainly the extent to which students can perform school procedures, e.g., to apply a known formula for some computation within a narrow formal context, which often imposes a particular solution method. Thus it remains unclear whether the students who succeed in these tests would present the same level of geometry knowledge in situations where the test format would not be similar to the way they have been taught.

In the present research a different method to measure visual and analytic reasoning was developed, based on the following considerations: First, we avoided setting our task in the typical geometry textbook style that could suggest deductive requirements and delimit visualization or measuring. Second, we did not impose a particular solution method to the solver but rather selected problems that could be solved using either visuo-spatial or analytic strategies, so that we could investigate spontaneous strategy choice. Third, we wanted to investigate not only whether students are able to use analytic strategies but also whether they are able to do so in situations that require them to inhibit visual-perceptual information processing and reason instead along formal geometric lines. Thus, a task was needed in which the perceptual difficulty of comparing shapes would be intensive and where the use of analytic strategies would lead to conclusions sometimes conflicting with visual-perceptual information.

The above theoretical considerations led to the development of the present Visual/Analytic Strategy Task (VAST). The VAST is a verbal/picture verification task. The participants are presented with a geometrical configuration that includes two shapes and are asked to decide whether a verbal statement that states that these shapes are congruent (Congruence domain), similar (Similarity domain), or occupy the same area (Area domain), is true or false. As shown in Figure 2, there are four types of configuration conditions in each geometrical domain: (a) the 'Appearance+/Reality+' condition where the two shapes both appear to be and are indeed congruent, similar or area equivalent; (b) The 'Appearance-/Reality-' condition where the two shapes neither appear nor are congruent, similar or area equivalent; (c) The 'Appearance+/Reality-' condition where the two shapes appear to be but are not congruent, similar or area equivalent; and (d) The 'Appearance-/Reality+' condition where the two shapes do not appear to be but are congruent, similar or

astronomy (Vosniadou, 2013; Vosniadou \& Brewer 1994). In geometry, the 'figural object' described by Fischbein (1993) to be formed from the synthesis of the 'perceptual' and 'conceptual' objects described by Batista (2007) is such a hybrid, synthetic conception. 


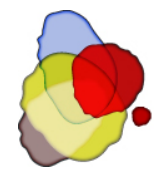

area equivalent. On the top of each configuration there is a verbal statement, such as, for instance, 'The lengths of the routes are the same' (See Figure 2, upper row). The participants are asked to decide whether this statement is true or false with respect to the geometrical configuration to which it refers.

In all three geometrical domains, the conditions (a) and (b) involve items purposely designed to be solved by visual estimation alone and which are consistent with the adoption of either a visual or an analytic geometric strategy (thereafter the VAST Consistent Subscale, or VAST-ConS). The conditions (c) and (d) are inconsistent with reliance on visual estimation alone and require for their correct solution reliance on geometrical knowledge and the adoption of analytic strategies (thereafter the VAST-InconS). The geometrical knowledge required involves either measurement and empirical confirmation or Euclidean deductive argumentation.

More specifically, in the case of the geometrical configurations a1 and a2 in Figure 2, the conclusions that the two routes are equal (in a1) and unequal (in a2) can be reliably achieved through visualspatial inspection. They can also be deduced on the basis of known geometrical properties: In a1, the conclusion of equality can be deduced from the congruence of the corresponding line segments, which are the opposite sides of the formed rectangles. In a2, the conclusion that Nick's path is shorter than John's path can be deduced from the geometrical axiom of triangle inequality - that the hypotenuse is always shorter than the sum of the right angled segments. In the case of the geometrical configuration a 3 and a4, however, the conclusions cannot be deduced by using visual strategies, but only through reliance on geometrical knowledge. In a3, in order to deduce the inequality of length line segments, one has to compute the hypotenuses of the formed right triangles and compare the oblique line segments with the vertical or horizontal ones. In a4 by drawing horizontal and vertical lines, the segments forming the zigzag "John's route" are equal to the corresponding segments forming the direct "Nick's route", as opposite sides of rectangles. The above rationale applies to all items of the test.

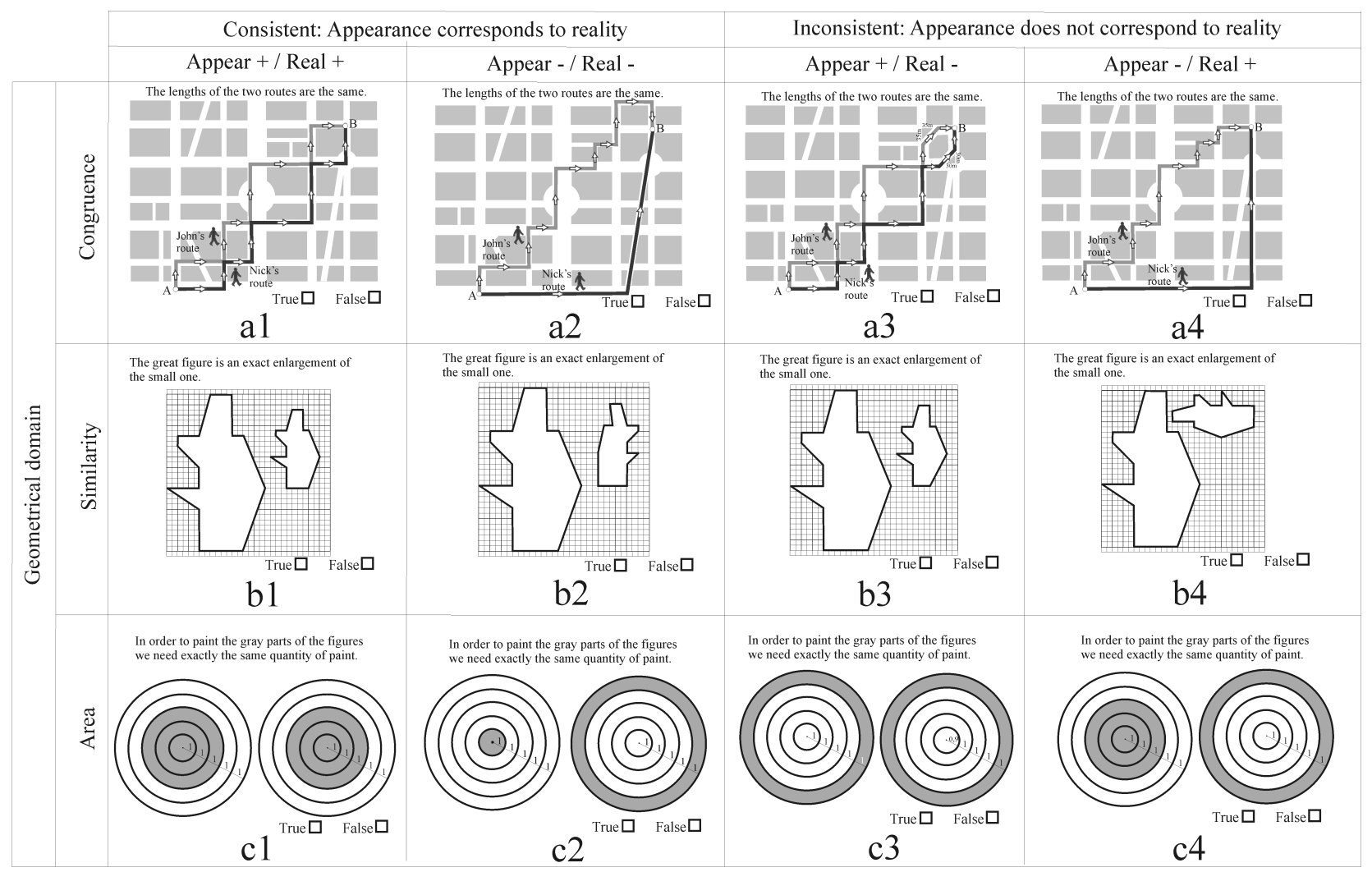

Figure 2. Sample items from the Visual/Analytic Shift Test (VAST). 


\subsection{Questions and Hypotheses of the Present Study}

Our purpose in the present study was to examine if the VAST is a reliable and valid test of visual and analytic reasoning in geometry. With respect to reliability, we wanted to find out whether Kuder-Richardson (K-R) reliability index was acceptable across the different sub-scales (Hypothesis 1).

With respect to validity, and in view of our theoretical position that the use of visual and analytic strategies is related to geometry expertise, we wanted to find out if the VAST would be able to differentiate the performance of experts in geometry from that of novices. For this reason, the VAST was administered to a group of mathematics teachers with extensive experience in teaching geometry and to a group of $11^{\text {th }}$ grade students who had been exposed to Euclidean geometry teaching. We hypothesized that if the VAST is a good test of the use of visual and analytic strategies, then the mathematics teachers should have high scores both in the Vast-ConS and in the VAST-InconS because of their expertise in geometry. On the contrary, the students would obtain high scores only in the VAST-ConS, which can be solved with visuo-spatial strategies and not in the VAST-InconS, which requires reliance on analytic strategies based on geometrical knowledge and the inhibition of visual estimation (Hypothesis 2).

The above hypotheses are different from what would be expected assuming that performance in the VAST is related only to individual differences in strategy use as opposed to geometry expertise. Individual differences in strategy use would not predict systematic differences in the performance of the high school students. Rather, some participants should do better in the visuo-spatial items and some others in the analytic items, regardless of their geometry knowledge (Hypothesis 3 ).

Hypothesis 4 concerned the relation between the VAST-InconS and geometrical knowledge, as measured by school grades in geometry (GG) and performance in a standardized test of geometrical knowledge (the California Standards Geometry Test - CSGT). High correlations were predicted between performance in the VAST-InconS, CSGT and GG, because they are all alternative measures of geometrical problem solving and geometrical knowledge.

Finally, we investigated the relation between VAST-IconS and two cognitive abilities that comprise spatial thinking: (i) abstract reasoning ability, i.e., the ability to identify patterns, analogical relationships and logical rules, and (ii) visuo-spatial ability - i.e., the ability to mentally visualize the rotation of objects. In view of the well-documented findings in the literature that spatial thinking is strongly related with students' performance in STEM subjects, we hypothesized that performance in the VAST-InconS should correlate positively with performance in the these two tests of spatial thinking (Hypothesis 5). However, in accordance with our theoretical position, namely that it is the acquisition of geometrical knowledge that leads to the use of analytic strategies, we expected that geometry knowledge (as measured by the CSGT) would significantly contribute to VAST-InconS performance, reducing the influence of the spatial thinking factor (Hypothesis 6).

\section{Method}

\subsection{Participants}

The participants included 30 mathematics teachers (age range 30-55 years, 18 men) and $13411^{\text {th }}$ grade students (age range 16.4-17.5 years, 71 boys). The mathematics teachers had considerable experience 
teaching high school geometry. The students were of middle-class backgrounds, came from two different schools, had four different geometry teachers, and were towards the end of a five-year course in geometry ${ }^{4}$.

\subsection{Materials}

The Visual Analytic Shift Test (VAST) consisted of four items for each geometrical domain counterbalanced across the four conditions described earlier. Thus, there were a total of 48 items ( 4 items for each geometrical domain $\times 3$ domains $\times 4$ conditions), randomly ordered.

The California Standards Geometry Test (CSGT) consisted of 15 items (5 for each geometrical domain) selected from the overall 96 items of the California Standards Geometry Test sample released in 2009 (http://www.cde.ca.gov/Ta/tg/sr/documents/cstrtqgeomapr15.pdf). The selection was made on the basis of the relevance of each item to the national geometry curriculum.

The Purdue Visualization of Rotations Test (ROT) is a test that determines how well one can visualize the rotation of three- dimensional objects. It is among the tests of spatial thinking less likely to be contaminated by analytical abilities. To restrict analytical processing, a time limit of 10 minutes for the 20item version of this test was strictly enforced (Bodner \& Guay, 1997).

The Abstract Reasoning Test (ART) is one of the tests of spatial thinking used by Wai et al., (2009). It is a non-verbal measure of fluid intelligence, consisting of 15 items.

School grades in Geometry were collected for all students participating in the study.

\subsection{Procedure}

The VAST was administered to the mathematics teachers individually in their school office. The VAST and the CSGT were administered to the students as a group test during a 45-minute class session. Their order of presentation was counterbalanced. The students were instructed to answer the VAST and CSGT items using whatever method they found suitable. Formulas were provided to students individually, if they asked for them. The ROT and ART were administered to small groups of students in the school computer lab. Completion of the electronic tests required approximately 30 minutes.

\section{Results}

\subsection{Reliability indices}

Since all measures were binominal, the Kuder-Richardson (K-R) reliability test was applied. The results showed that the reliability of the two subscales was acceptable (VAST-ConS, Kuder-Richardson (K$\mathrm{R})=.70$; VAST-InconS, Kuder-Richardson $(\mathrm{K}-\mathrm{R})=.75)$. Reliability of the rest scales are as follows: CSGT (Kuder-Richardson $(\mathrm{K}-\mathrm{R})=.76$, range of mean percentage performance: 13.33-100.00, Mean=63.82, $\mathrm{StD}=$ 22.35), ROT (Kuder-Richardson $(\mathrm{K}-\mathrm{R})=.74$, range: $1-20$, Mean= 8.59, $\mathrm{StD}=3.81$ ), and ART (KuderRichardson $(\mathrm{K}-\mathrm{R})=.57$, range: $5-15$, Mean $=9.89, \mathrm{StD}=2.49)$.

\footnotetext{
${ }^{4}$ The students had been taught the basic geometric concepts and methods based on empirical measurements and inductive generalizations in grades 7, 8, and 9. In grades 10 and 11 they were introduced to the procedures of deductive proofs characterizing Euclidean geometry.
} 


\subsection{Performance of the experts vs novices}

In order to examine the effect of the visual vs. analytic component on the participants' performance, two composite scores were computed: The mean percentage performances in the VAST Consistent Subscale (VAST-ConS) and in the VAST Inconsitent Subscale (VAST-InconS). Examination of the mean and standard deviation of the performance on the VAST-ConS, showed that the scale almost reached a ceiling effect (Mean Percentage $=83.86$, see Table 1). Thus, as was planned, this subscale consisted of easy items that could be successfully solved by the math teachers as well as by the students. Given that normality assumptions did not hold for this particular subscale, no parametric tests were applied.

Table 1

Means and StD as a function of Expertise and Item type (VAST-ConS vs. VAST-InconS) of the VAST

\begin{tabular}{|c|c|c|c|c|c|c|}
\hline \multirow[t]{3}{*}{ Participants } & \multicolumn{4}{|c|}{ Item Type } & & \\
\hline & \multicolumn{2}{|c|}{ VAST-ConS } & \multicolumn{2}{|c|}{ VAST-InconS } & \multicolumn{2}{|l|}{ Total } \\
\hline & Mean & StD & Mean & StD & Mean & StD \\
\hline Teachers & 92.000 & 7.575 & 78.841 & 11.685 & 85.392 & 7.525 \\
\hline Students & 81.637 & 12.454 & 50.751 & 15.314 & 66.175 & 10.312 \\
\hline Total & 83.857 & 12.321 & 56.770 & 18.606 & 70.293 & 12.566 \\
\hline
\end{tabular}

A t-test for independent samples was applied on VAST-InconS performance. Results showed significant difference between the two groups $[\mathrm{t}(138)=-9.324, \mathrm{p}<.001$, Mean $=50.75$, for the students, and Mean $=78.84$, for the math teachers]. The math teachers answered correctly almost all of the items in the VAST-InconS, whereas the 11th graders had considerable difficulty with the VAST-InconS. In agreement with Hypothesis 2, teachers' and students' performance was clearly differentiated in the VAST-InconS, where the performance of students was considerably lower than that of the teachers.

In order to examine Hypothesis 3, we plotted the students' individual mean percentage performance in the VAST-ConS and the VAST-InconS. Figure 3 shows the mean percent score of each participant on the y-axis. As can be seen, it was not the case that some participants performed well in the VAST-ConS and others in the VAST-InconS, as would have been predicted by the individual differences/cognitive style hypothesis. With very few exceptions, the items in the VAST-ConS that could have been solved using visuospatial strategies were much easier for each individual participant than the items in the VAST-InconS, which required recourse to analytic strategies. It can also be seen, that many students performed well only in the case of the VAST-ConS. 


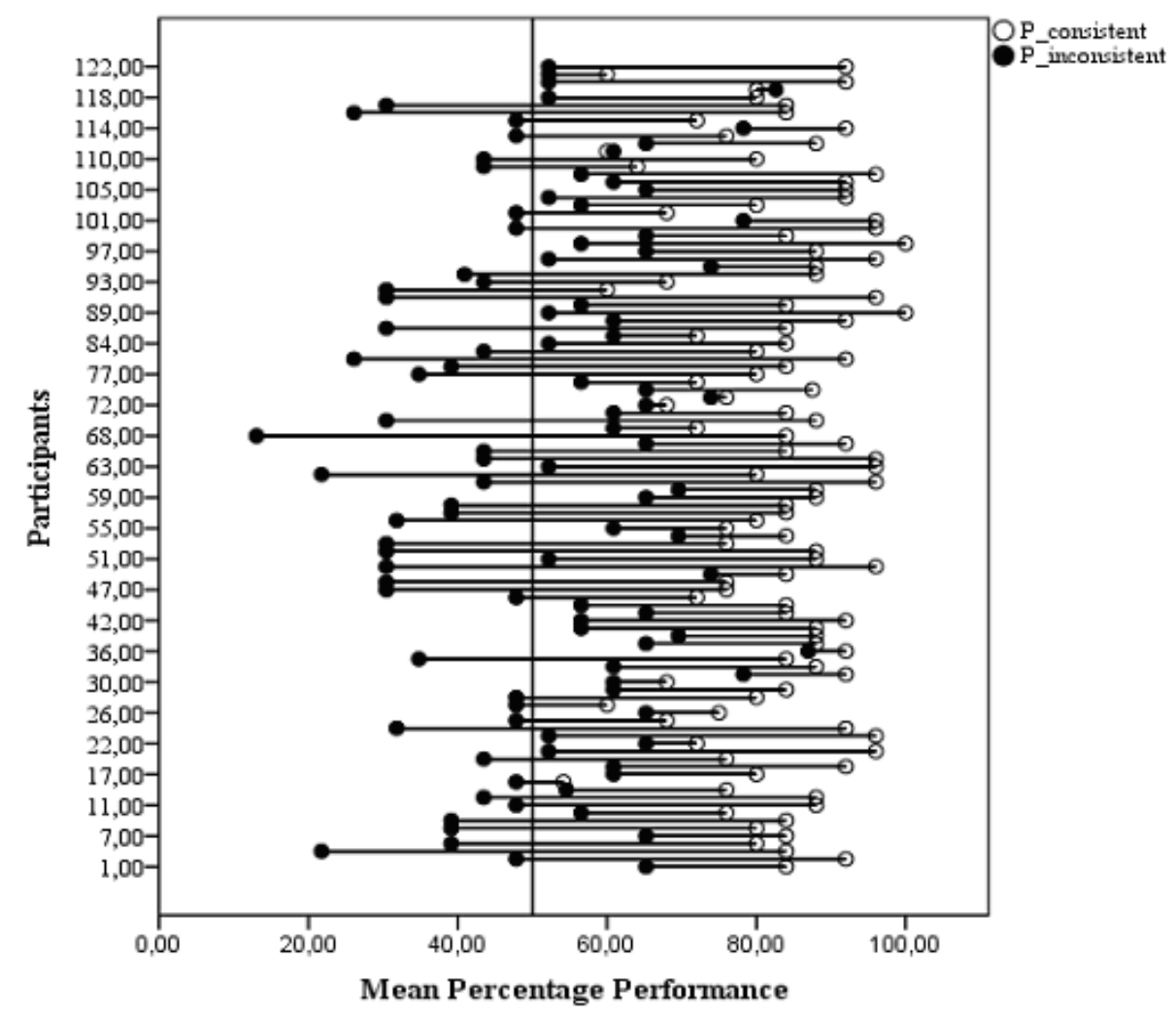

Figure 3. Individuals' mean performance in the VAST-ConS and the VAST-InconS. The performance of the teachers is shown above the horizontal line.

\subsection{Relations between the VAST-ConS, VAST-InconS CSGT, GG ROT and ART}

Due to the violation of the normality assumption of the VastConS, a Spearman's rho correlation analysis was performed on the mean scores of the VastConS with the VastInconS, CSGT, GG, ROT and ART. Results showed that VastConS correlated moderately only with ROT ( $\mathrm{rho}=.190, \mathrm{p}=.047$ ), whereas all other correlations were insignificant (with VastInconS, rho=.136, with CSGT, rho $=.165$, with GG, rho=.054 , and with ART, rho=.176).

\subsection{Relations between the VAST-InconS, CSGT and GG}

In order to examine Hypothesis 5, a correlation analysis (Pearson's r) was performed on the mean percentage scores on the VAST-ConS, the VAST-InconS, ROT (Cronbach alpha $=.74$, range: 1-20, Mean $=$ 8.59, $\mathrm{StD}=3.81$ ), and ART (Cronbach alpha= .57, range: 5-15, Mean=9.89, $\mathrm{StD}=2.49$ ). As predicted, the results showed statistically significant correlations between performance in the two VAST subscales, ROT and ART (Table 2).

\subsection{Relations between the VAST-InconS, ROT and ART}

In order to examine Hypothesis 5, a correlation analysis (Pearson's r) was performed on the mean percentage scores on the VAST-InconS, ROT (Cronbach alpha $=.74$, range: $1-20$, Mean= 8.59, $\mathrm{StD}=3.81$ ), and ART (Cronbach alpha= .57, range: $5-15$, Mean=9.89, $\mathrm{StD}=2.49$ ). As predicted, the results showed 
statistically significant correlations between performance in the two VAST subscales, ROT and ART (Table 2).

Table 2

Correlation between the measures of the study

\begin{tabular}{lccccc}
\hline & 1 & 2 & 3 & 4 & 5 \\
\hline 1. VAST-InconS & - & & & & \\
2. CSGT & $.429^{* *}$ & - & & & \\
3. GG & $.318^{* *}$ & $.575^{* *}$ & - & & \\
4. ROT & $.313^{* *}$ & $.357^{* *}$ & $.249^{* *}$ & - & \\
5. ART & $.366^{* *}$ & $.414^{* *}$ & $.285^{* *}$ & $.327^{* *}$ & - \\
**. Correlation is significant at the 0.01 level (2-tailed) & & & & \\
*. Correlation is significant at the 0.05 level (2-tailed) & & & &
\end{tabular}

A stepwise regression analysis was applied with the purpose of examining in greater detail the contributions of the above-mentioned measures of this study to VAST-InconS performance. The measures were inserted in the analysis in the following order: ROT, ART, and CSGT. The order of insertion followed the theoretical rationale of the present study, that is, general visuo-spatial ability (ROT) was inserted first, followed by general abstract reasoning ability (ART), and, finally, by performance on CSGT, which incorporated all the above and included geometrical knowledge. Based on our theoretical analysis, performance in CSGT should predict performance in the VAST-InconS best.

Table 3

Results of step-wise regression of ROT, ART, and CSGT on VAST-InconS

\begin{tabular}{lllllll}
\hline Model & & $\mathrm{B}$ & Std. Error & $\beta$ & $\mathrm{t}$ & Sig. \\
\hline 1 & ROT & 1.258 & .432 & .295 & 2.909 & .005 \\
\hline 3 & ROT & .901 & .430 & .211 & 2.096 & .039 \\
& ART & 1.772 & .581 & .307 & 3.048 & .003 \\
& ROT & .522 & .442 & .122 & 1.179 & .242 \\
& ART & 1.223 & .603 & .212 & 2.027 & .046 \\
& CSGT & .196 & .076 & .282 & 2.561 & .012 \\
& & & & & & \\
\hline
\end{tabular}


The results (see Table 3 ) showed that all three consecutive models had a good fit. For the first step (ROT) $[\mathrm{F}(1,89)=8.461, \mathrm{p}=.005]$, for the second step $(\mathrm{ROT}$ and ART) $[\mathrm{F}(2,88)=9.269, \mathrm{p}<.001]$, and for the third step (ROT, ART and CSGT) [F $(3,88)=8.755, \mathrm{p}<.001]$. As it can be seen in Table 3, when CSGT was entered in the model the contribution of ROT became non-significant $(\mathrm{p}=.242)$ and the contribution of ART became marginally significant $(\mathrm{p}=.046)$. These results fully confirmed Hypothesis 6 , indicating that geometrical knowledge, and not general visuo-spatial abilities and abstract spatial reasoning, accounted for the visual/analytic strategy shift as measured by the VAST-InconS.

In order to further validate the above result, a mediation analysis of the patterns of relations was applied on the data (see Figure 4), by using AMOS (version SPSS21) through bootstrapping (number of bootstrap samples $=2000$, Bias corrected confidence intervals $=.95$ ). First, the direct relations between ROT and ART on the VAST-InconS were computed. For ROT and VAST: two-tailed significance $\mathrm{p}<.045$, and for ART and VAST: two-tailed significance $\mathrm{p}<.019$. Thus, the results indicated that both paths were significant. We then tested two models, one with the CSGT and the other with geometry grades as mediating variables.

When the CSGT was added as a mediating variable, the indirect effect (i.e., the mediating path from ROT through CSGT to VAST-InconS) was significant $(\mathrm{p}=.006)$, and so was the mediating path from ART through CSGT to VAST-InconS $(\mathrm{p}=.001)$. Inspection of the direct effects showed that both relations were completely mediated by CSGT (for the path between ROT and VAST-InconS, $p=.143$ and for the path ART to VAST-InconS, $\mathrm{p}=.133$ ). The best model that resulted (see Figure 4), eliminating only the direct relation from ROT to the Vast-InconS, had an acceptable fit $\left(\chi^{2}(1)=2.384, p=.123, \mathrm{CFI}=.977\right.$, Standardized RMR $=$ $.04)$.

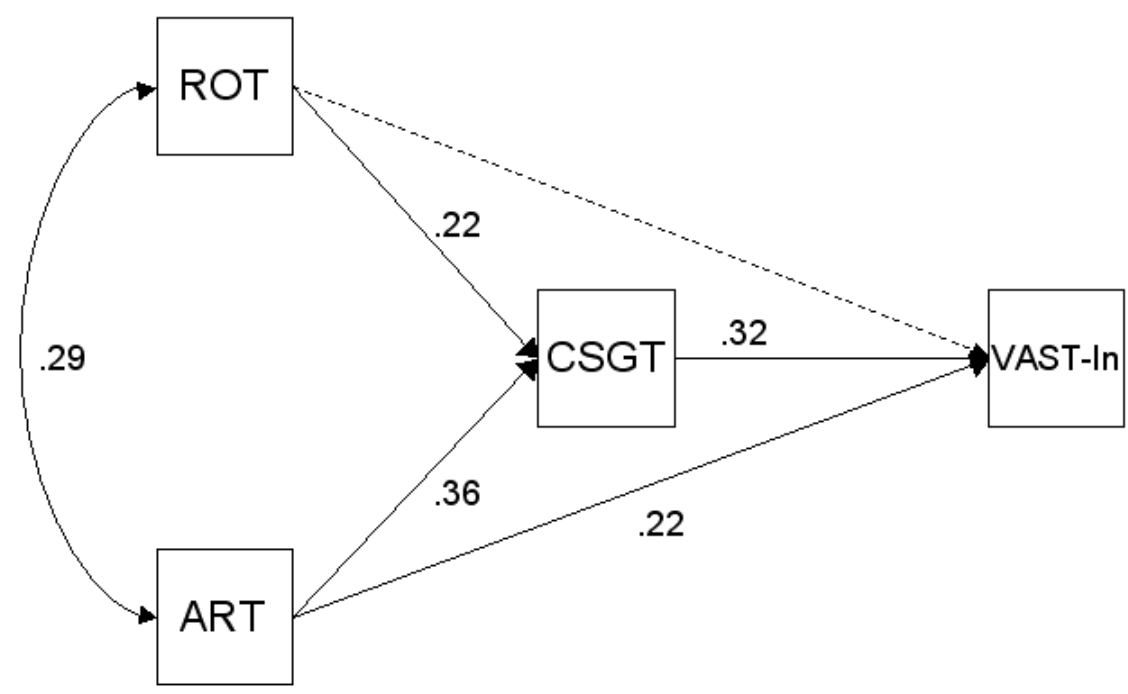

Figure 4. Regression weights of the Mediation Analysis between ROT, ART, CSGT, and VAST-InconS.

When geometry grades were treated as the mediating variable, the indirect effect from ROT through geometry grades to VAST-InconS was significant $(\mathrm{p}=.042)$, and so was the mediating path from ART through school grades to VAST-InconS $(p=.025)$. Inspection of the direct effects showed that the relation between ROT and the VAST-IconS was completely mediated by geometry grades (for the path between ROT and VAST-InconS $\mathrm{p}=.086$ ), whereas the relation between ART and the VAST-InconS was partially mediated by grades $(\mathrm{p}=.048)$. The final model, after eliminating the direct relation between ROT and the VAST-InconS did not, however, show a good fit $\left[\chi^{2}(1)=3.431, p=.06, \mathrm{CFI}=.942\right.$, Standardized RMR $=$ .05], since the value of $\chi^{2} / \mathrm{df}$ exceeded the value of 3 , and model's $\mathrm{p}$ value was statistically significant.

To conclude and summarize, the results of the mediation analysis confirmed the hypothesis that performance on the VAST-InconS will be mediated by geometrical knowledge, particularly when 


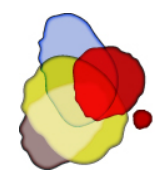

geometrical knowledge was measured by CSGT. In addition, the model with the best fit still retained the direct relation between analytic reasoning as measured by ART and the VAST-InconS.

\section{Discussion}

Our main purpose in this study was to develop and validate a task that could distinguish visuo-spatial from analytic reasoning in geometry. As mentioned in the introduction, there have been several attempts so far to develop tasks that capture the change from the visual to the descriptive/analytic level in geometry. These previous attempts were not very successful because they were based on the recall or recognition of definitions of shapes and their properties, did not require thinking based on relational properties, and/or did not require the application of formal geometrical thinking in novel situations.

The VAST differs substantially from these previous attempts because it avoids the typical geometrystyle problems that impose an analytical solution method and because it consists of tasks that can be solved using either visuo-spatial or analytic strategies. The greatest advantage of VAST, compared to previous tests, is that it focuses specifically on the antagonism between the two substantially different types of strategy in geometry and allows us to check individuals' abilities to spontaneously choose and adequately apply the correct strategy. Finally, the VAST investigates the ability to use analytic strategies in situations where the visual element plays quite a central role and where visual information processing must be inhibited in favour of formal geometrical thinking.

The results of the present study showed that the VAST consists of items that have good internal consistency. Most importantly the results show that the VAST is a valid test because it can differentiate geometry teachers from students and because it correlates highly with other tests of spatial thinking and geometrical knowledge.

\subsection{Are the differences in VAST performance related to geometry expertise?}

Despite small differences in the ease or difficulty of the solution of individual items, the main pattern of results was the same: The items in the VAST-ConS that could be solved correctly using visuo-spatial strategies were much easier for all participants than the items in the VAST-InconS which required the use of analytic strategies and the inhibition of the visual element.

As predicted (Hypothesis 2), the math teachers were able to solve both the VAST-ConS and the VAST-InconS. This result suggests that the math teachers have access to both visuo-spatial and analytic strategies. On the contrary, the 11th grade students could easily solve only the VAST-ConS, but had great difficulty with the VAST-InconS. This finding indicates that the students relied predominantly on visuospatial strategies and had difficulty in employing analytic strategies when required by the task, further confirming Hypothesis 2.

The finding that there were systematic differences in the performance of the math teachers and the students -- the math teachers performed well in both the VAST-ConS and the VAST-InconS, but the 11th grade students were able to perform well only in the VAST-ConS -- supports the argument that the use of analytic strategies is related to the acquisition of geometry expertise. As explained in the introduction, if the differences in VAST performance were due to individual differences in strategy use, then we should expect some mathematics teachers and some students to perform well in the VAST-ConS and others in the VASTInconS (Hypothesis 3). This was not however the case. The items in the VAST-InconS were systematically more difficult than the items in the VAST-ConS both for teachers and for students. Moreover, the students performed well only in the VAST-ConS, indicating that the majority of the students were able to successfully apply only simple visuo-spatial strategies. 
Additional evidence in favour of the interpretation that the use of analytic strategies requiring geometrical knowledge in the VAST-Inconsistent sub-scale comes from the results of the mediation analysis which showed that relations between performance in the ROT and the VAST- Inconsistent subscale was completely mediated by performance in the CSGT. This means that high performance in the VASTInconsistent scale requires not just domain-general analytic skills, but domain-specific geometrical knowledge and ability to use analytic thinking in a geometrical context.

The results showed considerable individual differences in the performance of the students in the VAST-InconS. These differences seem to be related to differences in geometry expertise within the student group. This conclusion can be deduced from the high correlations that were obtained between performance in the VAST-InconS, performance in the CSGT, and GG (Hypothesis 4). Students' performance in the VAST-ConS and assumed ability to apply simple visuo-spatial strategies did not correlate significantly with their GG or with their performance in the CSGT, confirming Hypothesis 4, namely, that this performance does not necessarily require geometrical knowledge.

\subsection{What is the contribution of the cognitive skills involved in spatial thinking in VAST}

\section{performance?}

The results of the present study showed high correlations between performance in the VAST-ConS and the VAST-InconS with both ROT and ART, confirming the prediction that spatial thinking as measured by tests of visuo-spatial and abstract (spatial) reasoning abilities contributes to students' performance in both scales of the VAST. However, performance in the VAST-InconS was also highly correlated with performance in the test of geometrical knowledge (the CSGT) and geometry grades. Most importantly the results of the stepwise regression confirmed that when the CSGT performance was inserted in the last step of analysis, the contribution of ROT and ART (which were statistically significant in the previous steps of the analysis), became non- or marginally significant. Furthermore, when a mediation analysis was applied on the data, the previously significant direct relations between performance in the VAST-InconS and ROT were mediated by the students' geometrical knowledge, as measured by performance in the CSGT. Since the direct relations between ART and VAST-InconS were not eliminated, however, it might be the case that analytic abilities are directly contributing to performance in the VAST-InconS.

To sum up, the results indicate that the correct use of analytic strategies cannot be explained only on the basis of visuo-spatial abilities and abstract reasoning, i.e., the cognitive skills that comprise spatial thinking, but requires the accumulation of considerable geometrical knowledge.

\subsection{Implications for a theory of geometrical thinking}

One of the main reasons we developed the VAST was in order to show that the learning of geometry requires significant conceptual changes to take place, and that instruction-induced conceptual changes culminate in the ability to use formal, geometrical knowledge in problem-solving and in the flexible use of visual/spatial and analytic strategies appropriate in the given contexts. The role of instruction here is quite crucial. As Fischbein (1993) stressed, "the development of figural concepts generally is not a natural process" (p. 161). The present findings support the hypothesis that the use of analytic strategies in geometry is not a matter of individual differences in cognitive style but a major intellectual achievement, a conceptual change, which requires the acquisition of new forms of geometrical thinking.

The application of analytic, formal geometrical knowledge in problem solving does not mean that visuo-spatial geometrical reasoning disappears. The fact that the math teachers could easily solve the items in the VAST-ConS suggests that they still had access to visual strategies. This issue needs to be investigated further, however, in view of the fact that analytic strategies could be used in the VAST-ConS also. Finally, a great deal more research is also required to investigate the hypotheses of the FT according to which the 


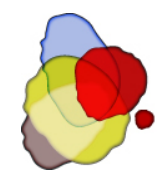

processes of acquisition of geometrical knowledge are slow and gradual rather than sudden or stage-like, and that these processes can give rise to fragmentation and synthetic models.

\subsection{Implications for learning and instruction}

The low performance of the students in the VAST-InconS after almost five years of instruction in geometry supports the argument that the systematic use of analytic strategies is a major intellectual achievement that requires considerable conceptual changes. It should be added here that the 11th grade students were in their $5^{\text {th }}$ year of geometry instruction, they had completed a two year course in plane Eucleadian Geometry, which was taught in a formal manner and accompanied by many geometry problems to be solved, and that they had an additional year's course in Analytic Geometry designed for students opting for university study in STEM subjects. Consequently, all the students had been taught the formulae and theorems required to answer correctly all the VAST items, both in the consistent and inconsistent sub-scales.

Thus, we can conclude from the above that it is possible to have acquired a great deal of school-type knowledge in geometry and not yet function at an analytic level in geometric thinking. Although geometry instruction focuses almost exclusively on the acquisition of formal geometrical knowledge and the application of analytic strategies, it does not seem to be very successful in transferring to situations different from the narrow school context in which it is taught and in producing the necessary conceptual changes. This situation is similar to what is happening in other STEM domains, such as physics or chemistry, where school instruction often fails to produce the necessary conceptual changes (e.g., Clement, 1982; diSessa, 1982). We hope that the present findings will further sensitize educators of the need to develop instruction that emphasizes not the recall and rigid application of formal definitions and rules but the constructive, dynamic activities of students that can help them understand how formal definitions fit with their visualspatial experiences and representations of geometrical shapes (Fischbein, 1993; Kilpatrick, Hoyles, Skovsmose, \& Valero, 2005; Lehrer Jenkins \& Osana, 1998).

\subsection{Limitations of the present study and future research}

The present work has a number of limitations and leaves several open questions to be answered by future research. First, the sample of the present study is small when attempting to validate a new measure such as the VAST and therefore the results presented in this exploratory study need to be replicated with larger samples and more age groups. In addition, reaction time studies as well as use of qualitative methods, such as interviews, think-aloud protocols and eye-movement tracking could be used to further validate the VAST.

Although performance in the VAST differentiates teachers from experts and is related to geometry expertise, the results do not provide information about how analytic reasoning in geometry actually develops. Developmental research is needed to further examine the hypotheses of the FT that knowledge acquisition in geometry is a continuous and not a stage-like process, during which fragmentation and synthetic conceptions are formed.

Future research needs to also investigate the contribution of intellectual abilities and mathematics knowledge to VAST performance by administering additional tests, such as a propositional test measuring verbal analytical skills, measures of visuo-spatial and phonological memory, speed of processing and executive function, as well as measures of mathematics abilities. Results from these tests could be used as co-variates in order to reduce as much as possible the effect of individual differences.

Last but not least, the relation between individual differences in spatial thinking and the use of visual and analytic strategies in geometry needs to be investigated further, preferably using longitudinal designs. If, as we claim, geometry expertise (and possibly expertise in other domains of STEM) requires the eventual development of analytic strategies, why are individuals who are good in spatial thinking more successful in STEM disciplines than those who are not? A possible explanation of this finding may be that individuals 
who are good in spatial thinking find it easier to do well in geometry early on, before conceptual changes in this domain require the development of analytic strategies based on formal, geometrical knowledge. Maybe because of these early successes, these students develop an interest in geometry (or other STEM-related disciplines), spend more time studying, and thus eventually undergo the conceptual changes and develop the analytic strategies required for geometrical expertise. This conjecture is consistent with the findings of the present study that spatial thinking as measured by visuo-spatial and abstract (spatial) reasoning tests contributes to success in the VAST, but is rendered insignificant for the VAST-InconS when geometrical knowledge is taken into account. This issue needs to be further researched.

\section{Keypoints}

- Considerable conceptual changes are required to go from visuo-spatial reasoning to analytic strategies in geometry

6. These changes are related to geometry expertise and not to individual differences

a. Questions arise about the role of visual-spatial reasoning in geometry expertise

\section{Acknowledgments}

The research reported in this paper is supported by a grant from the Greek Ministry of Education, General Secretariat for Research and Technology, MolVisEdu, THALIS - Aristotle University of Thessaloniki. We would like to thank Petros Roussos for helpful comments.

\section{References}

Battista, M. T. (2007). The development of geometric and spatial thinking. In F. Lester (Ed.), Second Handbook of Research on Mathematics Teaching and Learning, NCTM (pp. 843-908). Reston, VA: National Council of Teachers of Mathematics.

Bodner, G.M., \& Guay, R.B. (1997). The Purdue Visualization of Rotations Test. The Chemical Educator, 2(4), 1-17.

Burger, W. F., \& Shaughnessy, J. M. (1986). Characterizing the van Hiele levels of development in geometry. Journal for Research in Mathematics Education, 17, 31-48.

CSGT California Standards Test Geometry sample (2009). http://www.cde.ca.gov/Ta/tg/sr/ documents/cstrtqgeomapr15.pdf

Carey, S. (2009). The origin of concepts. New York, NY: Oxford University Press.

Cheng, Y.L., \& Mix, K.S. (2012). Spatial Training Improves Children's Mathematics Ability. Journal of Cognition and Development (published online, September 19, 2012). http://www.tandfonline.com/doi/pdf/10.1080/15248372.2012.725186

Chi, M. T. H., (2008). Three Types of Conceptual Change: Belief Revision, Mental Model Transformation, and Categorical Shift. In S. Vosniadou (Ed.), International Handbook of Research on Conceptual Change (pp. 61-82). New York, NY: Routledge.

Clement, J. (1982). Students' preconceptions in introductory mechanics. The American Journal of Physics, 50(1), 66-71.

Clements, D. H., \& Battista, M. T. (2001). Logo and Geometry. Journal for Research in Mathematics Education Monograph.Reston, VA: National Council of Teachers of Mathematics.

diSessa, A., (1982). Unlearning Aristotelian physics: A study of knowledge-based learning. Cognitive Science, 6(1), 37-75.DOI: 10.1207/s15516709 $\operatorname{cog} 0601 \_2$ 
Eisenberg, T., \& Dreyfus, T. (1991). On the reluctance to visualize in mathematics. In W. Zimmermann \& S. Cunningham (Eds.), Visualization in teaching and learning mathematics (pp. 26-37). Washington, DC: MAA.

Evans, J. St. B.T., \& Stanovich, K. (2013). Dual-process theories of higher cognition: Advancing the debate. Perspectives on Psychological Science, 8(3), 223-241. Doi: 10.1177/1745691612460685

Fischbein, E. (1993). The theory of figural concepts. Educational Studies in Mathematics, 24 (2), 139-162.

Gutiérrez, A., \& Jaime, A. (1998). On the assessment of the van Hiele levels of reasoning. Focus on Learning Problems in Mathematics, 20(2-3), 27-46.

Hegarty, M. (1992). Mental animation: Inferring motion from static diagrams of mechanical systems. Journal of Experimental Psychology: Learning, Memory and Cognition, 18, 1084-1102.

Hegarty, M., Montello, D. R., Richardson, A. E., Ishikawa, T., \& Lovelace, K. (2006). Spatial Abilities at Different Scales: Individual Differences in Aptitude-Test Performance and Spatial-Layout Learning. Intelligence, 34, 151-176.

Hegarty, M., \& Waller, D. (2006). Individual differences in spatial abilities. In P. Shah \& A. Miyake (Eds.), Handbook of Visualspatial Thinking. Cambridge, MA: Cambridge University Press.

Houdement, C., \& Kuzniak, A. (2003). Elementary Geometry Split into Different Geometrical Paradigms. Proceedings of CERME 3, Belaria, Italy. http://www.dm.unipi.it/ didattica/ CERME3/proceedings/Groups/TG7/TG7_Houdement_cerme3.pdf

Kastens, K.A., \& Ishikawa, T. (2006). Spatial thinking in the geosciences and cognitive sciences: A crossdisciplinary look at the intersection of the two fields. In C. A. Manduca \& D. W. Mogk (Eds.), Earth and mind: How geologists think and learn about the Earth (pp. 53-76). Boulder, CO: Geological Society of America.

Kilpatrick, J., Hoyles, C., \& Skovsmose, O. (Eds.) in collaboration with Valero, P. (2005). Meaning in Mathematics Education, USA: Springer.

Kozhevnikov, M., Motes, M., \& Hegarty, M. (2007). Spatial visualization in physics problem solving. Cognitive Science, 31, 549-579.

Lehrer, R., Jenkins, M., \& Osana. H. (1998). Longitudinal study of children's reasoning about space and geometry. In R. Lehrer \& D. Chazan (Eds.), Designing learning environments for developing understanding of geometry and space (pp. 137-167). Mahwah, NJ: Erlbaum.

Lynn, B. M., \& Lynch, C. M. (2010). van Hiele Revisited. Mathematics Teaching In The Middle School, 16(4), 232-238.

Newcombe, N. S. (2010). Picture this: Increasing math and science learning by improving spatial thinking. American Educator, Summer, 29-43.

Newcombe, N. S., \& Frick, A. (2010), Early education for spatial intelligence: Why, what, and How. Mind, Brain and Education, 4(3), 102-111.

Newcombe, N. S., \& Huttenlocher J. (2000). Making space: The development of spatial representation and reasoning. Cambridge, MA: MIT Press.

Newcombe, N. S., Uttal, D. H. \& Sauter, M. (in press). Spatial Development. In P. Zelazo (Ed), Oxford handbook of developmental psychology. New York, NY: Oxford University Press.

Piaget, J., \& Inhleder, B., (1948/1967). The child's conception of space. London: W.W. Norton \& Company.

Piaget, J., Inhelder, B., \& Szeminska, A. (1948/1960). The child's conception of geometry. London: Routledge and Kegan Paul.

Pitta-Pantazi, D., \& Christou, C. (2009). Cognitive styles, dynamic geometry and measurement performance. Educational Studies in Mathematics, 70, 5-26.

Sanchez, C.A. (2012). Enhancing visuospatial performance through video game training to increase learning in visuospatial science domains. Psychonomic Bulletin \& Review, 19 (1), 58-65.

Schwartz, D.L., \& Black, J.B. (1996). Shuttling between depictive models and abstract rules: Induction and fallback. Cognitive Science, 20, 457-497.

Shea, D. L., Lubinski, D., \& Benbow, C. P. (2001). Importance of assessing spatial ability in intellectually talented young adolescents: A 20-year longitudinal study. Journal of Educational Psychology, 93(3), 604-614. 
Shtulman, A., \& Valcarcel, J. (2012). Scientific knowledge suppresses but does not supplant earlier intuitions. Cognition, 124, 209-215.

Spelke, E. S., \& Kinzler, K. D. (2007). Core knowledge. Developmental Science, 10, 89-96.

Spelke, E.S., Lee, S. A., \& Izard, V. (2010). Beyond core knowledge: Natural geometry. Cognitive Science, 34(5), 863-884

Stieff, M. (2007). Mental rotation and diagrammatic reasoning in science. Learning and Instruction, 17, 219234.

Usiskin, Z. (1982). Van Hiele levels and achievement in secondary school geometry. Colombus, OH: ERIC.

Uttal, D. H., Meadow, N. G., Tipton, E., Hand, L. L., Alden, A.R., Warren, C., \& Newcombe, N.S. (2013). The malleability of spatial skills: A meta-analysis of training studies. Psychological Bulletin, 139, 352402.

Van Hiele P. M. (1986). Structure and Insight: A theory of Mathematics Education. London: Academic Press Inc.

Vosniadou, S., \& Brewer, W. F. (1994). Mental Models of the Day/Night Cycle. Cognitive Science, 18, 123183.

Vosniadou, S. (2013). Conceptual change in learning and instruction: The framework theory approach. In S. Vosniadou (Ed.), International Handbook of Research on Conceptual Change, $2^{\text {nd }}$ Edition (pp. 11-30). New York, NY: Routledge.

Vosniadou, S., \& Skopeliti, I. (2014). Conceptual change from the framework theory side of the fence. Science and Education, 23(7), 1427-1445.

Vosniadou, S., Pnevmantikos, D., Makris, N., Ikospentaki, K., Lepenioti, D., Chountala, A., \& Kyrianakis, G. (2015). Executive Functions and Conceptual Change in Science and Mathematics Learning, $7^{\text {th }}$ Annual Conference of the Cognitive Science Society, Pasadena, CA. https://mindmodeling.org/cogsci2015/papers/0434/paper0434.pdf

Wai, J., Lubinski, D., \& Benbow, C. P. (2009). Spatial ability for STEM domains: Aligning over 50 years of cumulative psychological knowledge solidifies its importance. Journal of Educational Psychology, 101(4), 817-835.

Wiser, M., \& Smith, C. (2013). Learning and teaching about matter in the middle school years. How can atomic-molecular theory be meaningfully introduced? In S. Vosniadou (Ed.), International Handbook of Research on Conceptual Change, $2^{\text {nd }}$ Edition (pp. 177-194). New York, NY: Routledge.

Zazkis, R., Dubinsky, E., \& Dautermann, J. (1996). Coordinating visual and analytic strategies: A study of students' understanding of the group D 4. Journal for Research in Mathematics Education, 27(4), 435457. 